Article

\title{
Rapamycin Alleviated the Symptoms of Fat Embolism Syndrome by Modulating Autophagy in a Rat Model
}

\author{
Ying Zhang, Rong Zhang, Xiaotao Xu, Aizhong Wang ${ }^{1 *}$ \\ ${ }^{1}$ Department of Anesthesiology, Shanghai Sixth People's Hospital East Affiliated to Shanghai \\ University of medicine \& Health Sciences.
}

\section{${ }^{*}$ Corresponding author}

Address correspondence to: Aizhong Wang, Shanghai Sixth People's Hospital East Affiliated to Shanghai University of medicine \& Health Sciences, NO. 222, huanhuxisan Road, Shanghai 200233, China; Tel: 0086-21-38297723; Fax: 0086-21-38297724; E-mail: w19680420@sohu.com

The work was done at Shanghai Sixth People's Hospital East Affiliated to Shanghai University of medicine \& Health Sciences

The authors declare no potential conflicts of interest.

Acknowledgments:

The authors thank the instruction of prof. Aizhong Wang and acknowledge the contribution of all investigators at all participating study sites. This study was supported by SUMHS seed foundation project (No. HMSF-17-21-017) and grants from the National Natural Science Foundation of China (No. 81772062). 


\section{Abstract}

As a high fatal disease, fat embolism syndrome is complication, which brought great pain to patient and their family served as a serious social burden. The mechanism of FES remains unclear. Autophagy controls the cell survival and homeostasis by removing the mis-folded proteins and damaged organelles as well as intracellular pathogens through a lysosomal degradation pathway. Increasing research documented that autophagy was wildly involved in variety of human diseases and had huge therapeutic potential. In our study, we first established the rat model of FES with the liquid fat by tail vein injection. We observed the up-regulated MPO expression and activity, increased Wet-to-Dry (W/D) lung weight, promoted quantity of fat granules, and the dominant disorder in the lung rat model of FES, compared to the control group. These observations demonstrated that we successfully build the rat model of FES. Then, we sought to dissect the role of the autophagy in the rat model of FES. The western blots analysis showed that the autophagy was inhibited in the rat model of FES constructed with liquid fat. Furthermore, Rapamycin could restore the repression of autophagy in rat model of FES. These investigations illustrated that autophagy was involved in FES. In addition, our experiments showed that Rapamycin could alleviate the symptoms of FES. Taken together, our study demonstrated the participation of autophagy in FES and further, as a potential therapeutic target, the modulation of autophagy could affect the symptom of rat model of FES.

\section{Key words}

fat embolism syndrome (FES), autophagy, rapamycin, rat model 


\section{Introduction}

Fat embolism syndrome (FES), defined as fat within the circulation, happen after trauma, surgery and fat emulsion input and in other clinical conditions [1-3]. Documents reported that FES often occurred in non-traumatic patients, such as acute pregnancy fatty liver, heat stroke, alcoholism, sickle red blood cell anemia, liver necrosis and diabetes [4-6]. Clinically, FES can lead to serious pulmonary injury, such as ARDS, pulmonary hypertension, right heart failure, arteriovenous shunt and alveolar hypo-perfusion, interstitial hemorrhage, edema, chemical pneumonia and death $[3,6,7]$. Nowadays, although various methods were employed for treatment of clinical FES, such as early fixation, injury control orthopedics (IVO), extracorporeal membrane lungs and drugs, but the mortality rate still up to $20 \%[8,9]$. Research reported that several animal models for FES were constructed to investigate the mechanism of FES, but the mechanisms of FES remain largely unclear [10-14].

Autophagy, derived from the Greek word meaning "self-eating", was a basic and fundamental cellular process for the degradation of cytoplasmic components (proteins, lipids, organelles, etc.) $[15,16]$. As the number of scientists investigating autophagy has increased, the complexity of autophagy has become increasingly dissected. There are three dominant types of autophagy have been described: macroautophagy, microautophagy, and chaperonemediated autophagy [17]. Autophagy is a dynamic biological process that consists of the initiation stage, the extension phase, the mature stage, and the degradation phase, and is markered by a series of autophagy-related genes (Atg), such as LC3, Beclin 1, ULKI, ATG5 and ATG7 [18-20]. p62 plays an important role in the formation of autophagosomes and p62 reduction indicates a sign of autophagic clearance. Mammalian target of Rapamycin (mTOR), a serine / threonine protein kinase, is one of the important targets of cell signaling pathway and is known as an autophagy protein. Studies have shown that PI3K / AKT / mTOR / p70S6K signal pathway could negatively regulate the autophagy. Document reported that autophagy play an important role in controlling cell development and differentiation, tissue homeostasis and diseases, such as cancer, acute brain injury neurodegeneration disease and aging[21,22].

Recently, several reports reported that inflammatory response is associated with FES [2325]. FFA and triglycerides can increase the release of interleukin and chemokines and further, exacerbated the inflammatory response and led to interstitial hemorrhage, edema, chemical 
pneumonia $[7,26]$. Recently, increasing documents investigated the role of autophagy in both innate and adaptive immunity and the influences exerted on the pathogenesis of inflammatory diseases $[27,28]$. Work in Devendrak Agrawal' lab demonstrated that TNF- $\alpha$ could promote the expression of the autophagy genes LC3 and Beclin 1 through activation of the Jun kinase signaling pathway [29]. Thus, it reported that both the FES and autophagy could participate in the inflammatory response.

In our study, we employed the liquid fat to build the rat model of FES successfully. Then, we examined the role of autophagy in the rat model of FES and found that autophagy was repressed in the rat model of FES and the restoration of autophagy by Rapamycin could alleviate the symptoms of FES. Thus, we dissect the relations between autophagy and FES, which provides a basis for further study of the FES mechanism. 


\section{Materials and Methods}

\section{Animal model and Rapamycin administration}

All animal experiments were conducted under the guidelines described in "Principles of Laboratory Animal Care" (National Institutes of Health publication No. 85-23, revised 2011) and approved by the ethical committees of the 6th People's Hospital affiliated Shanghai Jiaotong University (SYXK (Shanghai, China) 2011-0128, 1 January 2011).

Wistar rats (10 weeks old, male, weight $(250 \pm 20 \mathrm{~g}))$ used in this study were obtained from the Shanghai Sippr-Bk laboratory Animal Co. Ltd.(Shanghai, China). All rats were fed with standard laboratory and tap water and housed in humidity and temperature-controlled ventilated cages on a 12-h light/dark cycle. Fifty rats were randomly divided into control and fat embolism groups $1-4$ ( $n=10$ per group). The FES rats were constructed with allograft perirenal fat of rats $(0.706 \mathrm{~mL} / \mathrm{kg}$ body weight) by tail vein injection. Then rats were intravenously injected with the same volume of sterile saline as the control group. Lung tissue was obtained 1, 6, 12 and $24 \mathrm{~h}$ after fat injection. Sodium Rapamycin (>95\% pure) was purchased ( Sigma, St. Louis, MO, USA ) and dissolved in $0.9 \% \mathrm{NaCl}$ solution containing $1 \%$ DMSO (ABCONE, Shanghai) according to the manufacturer's instructions. Rapamycin was administrated by intraperitoneal injection at a dosage of $2 \mathrm{mg} / \mathrm{kg}$ (body weight) $30 \mathrm{~min}$ before fat injection.

\section{Measurement of Wet-to-Dry (W/D) Lung Weight}

The animals were euthanized by $5 \%$ chloral hydrate and the lung tissue was excised. After wiping the surface blood, the wet weight $(\mathrm{W})$ of obtained tissues were measured and then dried in the oven at $60^{\circ} \mathrm{C}$ for $72 \mathrm{~h}$. The dry weight (D) is got and the W/D value is calculated.

\section{Hematoxylin-eosin (HE) staining}

All animals were humanely sacrificed and lung tissues were embedded in $4 \mu \mathrm{M}$ paraffin sections. HE staining was performed to as described previously [30]. In short, slides for HE staining were immersed in alcohol at different concentration. Then, slides were immersed in a Harris hematoxylin liquid and $1 \%$ eosin alcohol under the corresponding conditions. The morphological structure of the rats' lung were observed and recorded under Olympus $C K X 53$ microscope.

\section{Oil red staining}

Oil red O staining was performed according to the method [31]. The entire lung of the rats 
were cut open and stained with Oil Red O for $30 \mathrm{~min}$ and then washed with ethanol 3 times. The fat accumulated in lung tissues observed and recorded under Olympus CKX53 microscope. Total RNA isolation, RT-PCR, qRT-PCR

Total RNA were isolated the lung of each rat as described above using TRIzol (Invitrogen, Carlsbad, CA, USA) according to the manufacturer's instructions. The RNA concentration was detected using a Nano Drop ND-1000 spectrophotometer (Thermo Scientific). Total RNA (1 $\mu \mathrm{g})$ was used for first-strand cDNA synthesis using the Primer Script RT reagent kit with gDNA Eraser (TaKaRa). Quantitative real-time RT-PCR analysis was performed to determine the levels of MPO in lung tissues with the ABI PRISM 7500 Fast Real-Time PCR System (Applied Biosystems) and the SYBR Green mix (TaKaRa). The primers for MPO were: forward primer F; 5'-CACTGGCATCACTACCGTGT-3', and reverse primer R; 5'- CCAATGGCCTCCGTTCCTC3'. The primers for GAPDH were: forward primer F; 5'- CATGAGAAGTATGACAACAGCCT-3', and reverse primer R; 5'- AGTCCTTCCACGATACCAAAGT-3'. The data were analyzed by the 2- $\triangle \triangle \mathrm{CT}$ method, and GAPDH served as loading control.

\section{Western blot analysis}

Western blot analysis was operated as described previously [32]. The protein was extracted using RIPAlysis buffer and separated by SDS polyacrylamide gel electrophoresis (PAGE) and transferred onto polyvinylidene fluoride membranes (Millipore, Billerica, MA). All antibodies were performed according to the manufacturer protocol. Anti-SQSTM1 / p62 antibodies (ab109012), anti-mTOR antibodies (ab32028), anti-P70S6 Kinase alpha antibodies (ab186753), anti-P70S6 Kinase alpha antibodies (ab59208) were purchased from Abcam (Cambridge, British). The anti-GAPDH antibodies (Rabbit, DH0261\#) and Enhanced Chemiluminescence Substrate (DH0101) were obtained from Donghuan Biotech Co., Ltd (Shanghai, China). Proteins were visualized using enhanced chemiluminescenceand analyzed using Gelpro Analyzer Software.

\section{Statistical analyses}

Data were analyzed by two-tailed Student's t-test and expressed as mean \pm standard deviation (S.D.) of triplicates. P-values less than 0.05 were considered statistically significant. The symbol * denotes statistical difference $(p<0.05)$, while ** represents great significant difference $(p<0.01)$ in a two-tailed Student's t-test. 


\section{Results}

\section{Construction of the rat model of FES using allograft perirenal fat of rats}

Recently, a variety of FES models of were established with different methods. In our study, we established the rat model of FES using allograft perirenal fat of rats at the dose of 0.706 $\mathrm{mL} / \mathrm{kg}$ body weigh by tail vein injection and employed variety of experiments to dissect the establishment of FES models, such as the qPCR assay, HE staining assay, measurement of Wet-to-Dry (W/D) Lung Weight and Oil red staining. The results of the qPCR and activity analysis demonstrated that the MPO expression and activity were up-regulated at 6, 12 and 24 hr $(p<0.05)$ in the FES group compared with the control group (Fig. 1A and B). Further, W/D measurement showed that the lung W/D ratio was increased at 16,12 and $24 \mathrm{hr}(p<0.05)$ in the FES group (Fig. $1 \mathrm{C}$ ). The results of HE staining assay showed great pulmonary edema with a widened interstitial space, ruptured alveolar wall and alveolar infiltration of inflammatory cells in the FES group (Fig. 1 D). In addition, the oil red staining analysis illustrated that the elevated quantity of fat granules in the FES group (Fig. $1 \mathrm{E}$ ). Taken together, these results demonstrated that we established the rat model of FES using allograft perirenal fat of rats. 

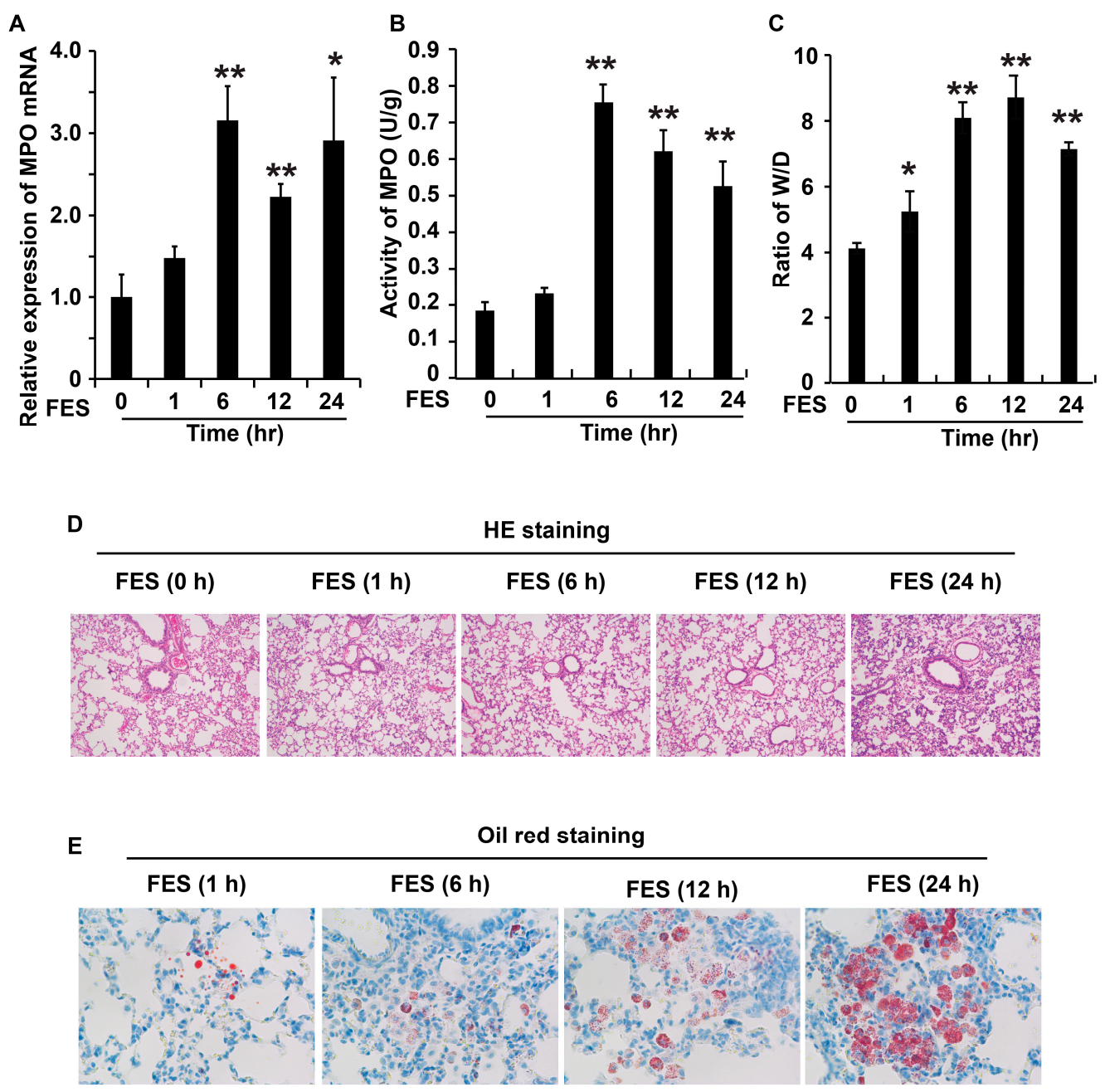

Figure 1. Construction of the rat models of FES using allograft perirenal fat of rats.

(A) qPCR assay analysis of MPO expression in rat model of FES treated with allograft perirenalat different time: $0 \mathrm{hr}, 1 \mathrm{hr}, 6 \mathrm{hr}, 12 \mathrm{hr}, 24 \mathrm{hr}$, respectively. GAPDH was used as control. (B) MPO activity assay analysis of MPO activity at different time. (C) W/D measurement analysis of the lung W/D ratio in the FES group. (D) HE staining analysis of the disorder in the lung of FES models. (E) Lung sections from the FES groups were stained with Oil red O. Each experiment was repeated a minimum of three times and the symbol * denotes a statistically significant difference $(p<0.05)$, while ** represents a highly significant difference $(P<0.01)$ in a two-tailed Student's t-test.

\section{Autophagy was involved in the model of FES}

Recently, increasing researches reported that autophagy exerts an important role in variety of human diseases and have the potentiality to be serving as novel therapeutic targets. However, there is no investigation to dissect the relationship between autophagy and FES so 
far. Thus, in this study, we sought to explore the role of autophagy in FES.

To explore whether autophagy was participated in the pathogenesis of FES, we applied western blot assay to detect the biological markers of autophagy, such as p62, mTOR, p72 and their phosphorylated types. The results of western blot analysis demonstrated that the expressions of p-mTOR, p-p72 and p62 were up-regulated at 1, 6, 12 and $24 \mathrm{hr}(p<0.05)$ compared to the control group (Fig. 2A). These results demonstrated that autophagy was inhibited in the FES group. To further confirm of the participation of autophagy in FES, we treated the rats with Rapamycin, which was known as an activator of autophagy by intraperitoneal injection. The results of western blots assay illustrated that Rapamycin could inhibit the increased expressions of p-mTOR, p-p72 and p62 in the FES group treated with Rapamycin compared to the FES group (Fig. 2B). Our study demonstrated that autophagy was involved in the pathogenesis of FES. 

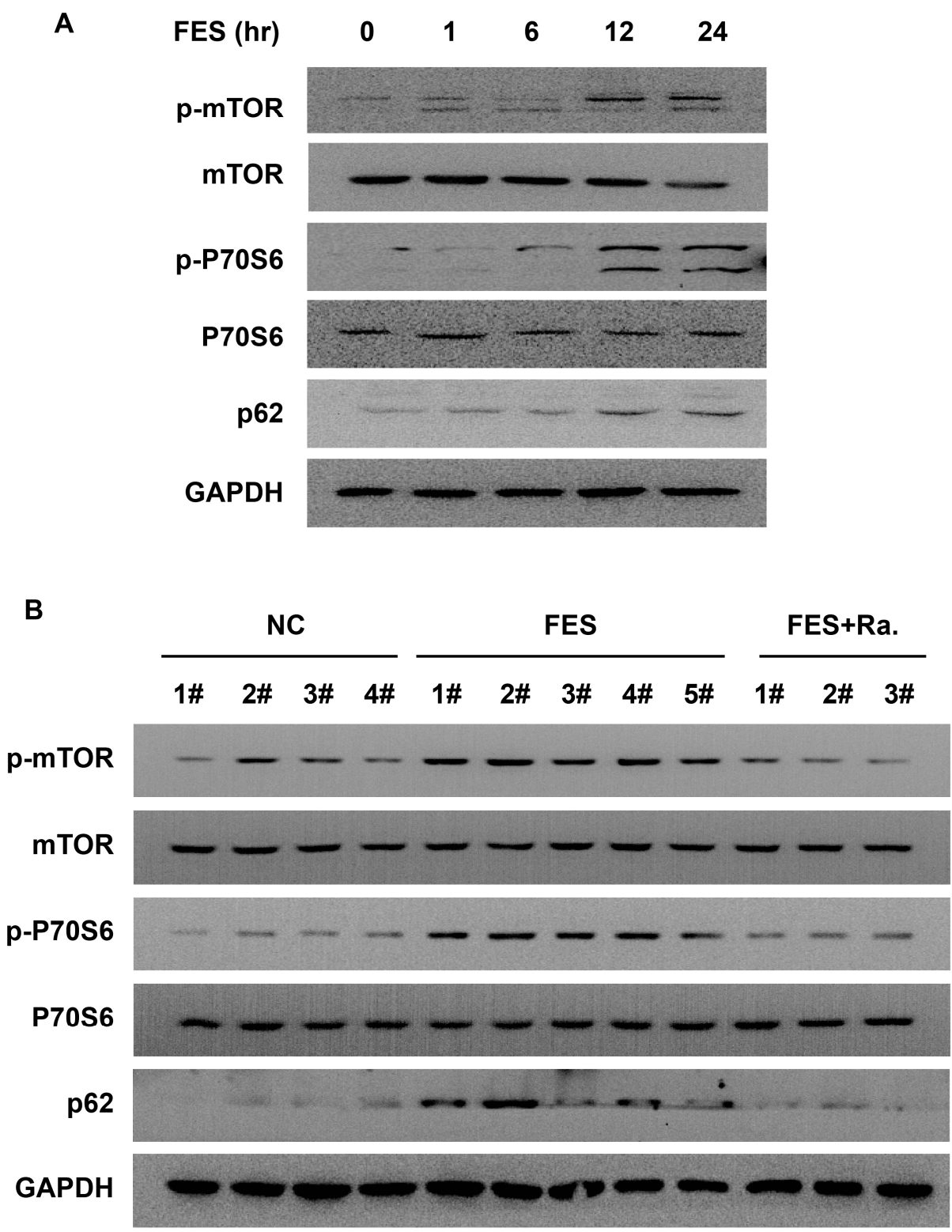

Figure 2. Autophagy was inhibited in the rat model of FES.

(A) Western blot analysis of biological markers of autophagy, such as p62, mTOR, p72 and their phosphorylated types in rat model of FES treated with allograft perirenalat different time: Ohr, $1 \mathrm{hr}, 6 \mathrm{hr}, 12 \mathrm{hr}, 24 \mathrm{hr}$, respectively. GAPDH expression was measured as control. (B) Western blot analysis of biological markers of autophagy in rat model of FES treated with or without Rapamycin. GAPDH expression was measured as control. Each experiment was repeated a minimum of three times.

\section{The modulation of autophagy could alleviated the symptoms of FES}

FES brings great pain to patient and their family and serves as a serious social burden, and more seriously, the mortality of FES was up to $36 \%$. Nowadays, early fixation, injury control 
orthopedics (IVO), extracorporeal membrane lungs and drugs were employed in the therapy of FES. Consideration of the high mortality rate of FES, there is a need to find a better way to treat FES. Results in our study have demonstrated that autophagy was involved in FES.

To dissect autophagy mode of action, we examined the role of FES in models treated with Rapamycin. The results of the qPCR and activity analysis demonstrated that the up-regulation of MPO expression was repressed $(p<0.01)$ in the FES group treated with Rapamycin (Fig. 3A and $B$ ). Furthermore, the results of W/D measurement showed that the lung W/D ratio was decreased $(p<0.01)$ in the FES group treated with Rapamycin (Fig. $3 \mathrm{C}$ ). In addition, the HE staining assay showed that the damaged structure of lung tissue was alleviated by Rapamycin (Fig. 3 D). Taken together, our results illustrated that Rapamycin could alleviated the symptoms of FES. 

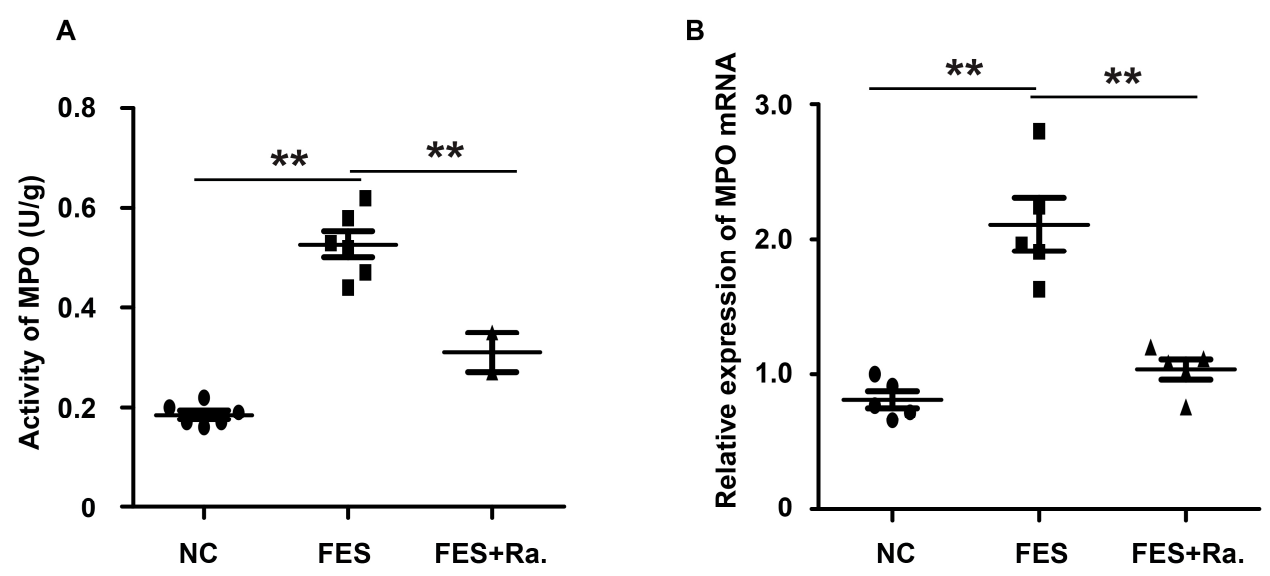

C

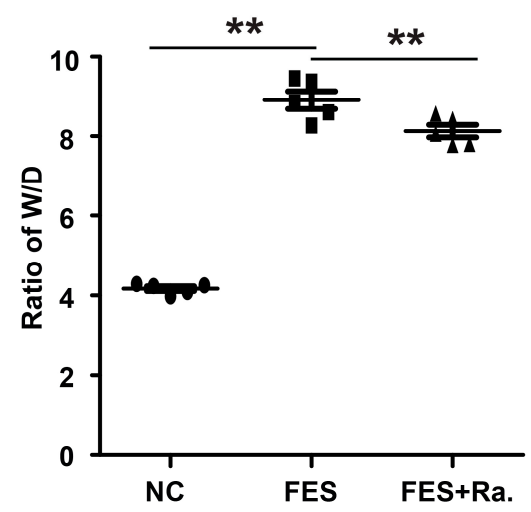

D

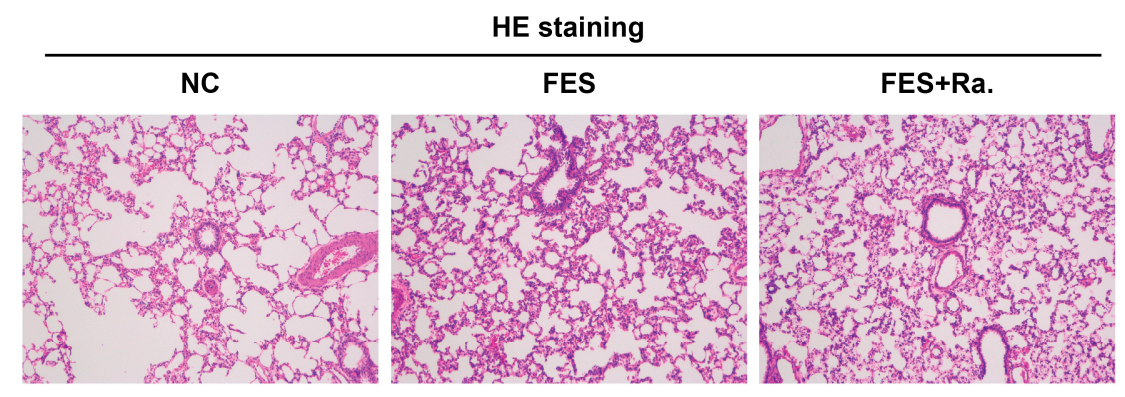

Figure 3.The modulation of autophagy could alleviate the symptoms of FES.

(A) MPO activity assay analysis of MPO activity in rat model of FES treated with or without Rapamycin. (B) qPCR assay analysis of MPO expression in rat model of FES treated with or without Rapamycin. GAPDH was used as control. (C) W/D measurement analysis of the rate of W/D of the lung of rat model of FES treated with or without Rapamycin. (D) HE staining analysis of the disorder in rat model of FES treated with or without Rapamycin. Each experiment was repeated a minimum of three times and the symbol * denotes a statistically significant difference $(p<0.05)$, while ${ }^{* *}$ represents a highly significant difference $(p<0.01)$ in a two-tailed Student's t-test. 


\section{Discussion}

Reports documented that the incidence of FES ranges from 2 to $22 \%$ and the mortality various from 10 to $36 \%$ [33-35]. Thus, FES, as a serious and a potentially life threatening complication, caused more and more attention of clinicians and researchers. There are variety of incentives to cause FES, such as trauma, surgery and fat emulsion input, heat stroke, liver necrosis, alcoholism, acute pregnancy fatty liver, sickle red blood cell anemia and diabetes. Currently, more and more different animal models of FES were established with different drugs and methods in various of animals, such as neutral fat in rats, lipopolysaccharide (LPS) in rats, triolein in cats, surgical approach in dogs and rabbit [11-13,36-38]. In this study, we build the rat models of FES using allograft perirenal fat by tail vein injection. Then, we evaluated the symptoms of the model. We found that the quantity of fat, MPO expression and W/D ratio of lung weight of models were both increased (Fig. 1A, B, C and E). Furthermore, the structure of the lung in FES models was destroyed (Fig. 1 D). These results demonstrated that we successfully established the FES models in rats. However, consideration of the variety of incentives for FES, there still need more methods to construct FES models that are much close to specific clinical symptoms of FES.

As a potentially life threatening complication, FES bring great pain to patient and their family and serve as a serious social burden. However, the mechanism of FES was still vague. There are two dominant mechanisms for FES. One is that mechanical embolism theory, and the other is biochemical damage theory. In short, mechanical embolism theory was thought that the fat particles in the venous circulation resulting the vascular blockage, pulmonary embolism and further, pulmonary hypertension, right heart failure, arteriovenous shunt and alveolar hypoperfusion. Biochemical damage theory believed that fat embolus in blood format glycerol and free fatty acids (FFA) and resulted the diffuse interstitial pneumonia, acute pulmonary edema, amplified inflammatory response and more serious, interstitial bleeding, edema, chemical pneumonia $[3,7]$. Thus, we are urgent to further investigate the real comprehensive mechanism of FES.

As an evolutionarily conserved catabolic process, autophagy has attracted more and more scientists' attention over the past two decades. Documents demonstrated that autophagy contributes to cell survival and homeostasis by removing them is folded proteins and damaged 
organelles as well as intracellular pathogens through the lysosomal degradation pathway $[16,39]$. However, autophagy is a complicated pathway and activated by variety of factors, such as exposure to anoxia, starvation, chemical agents and radiation. And autophagy is involving multiple proteins, such as class PI3K and Atg complexes, Beclin-1, LC3 proteins [40,41].

Consideration of the progression of our understanding of autophagy in mechanistic and pathophysiological diseases over the past decade, the role of autophagy and autophagyrelated diseases and a plethora of other diseases become increasingly clear. Documents reported that autophagy was wildly involved in variety of human diseases, such as cancer, inflammatory diseases, Parkinson disease, cardiovascular Disease, diabetes mellitus [42-45]. However, the relation of autophagy in many human diseases remains largely unexploited. In our study, we found that autophagy in FES was inhibited (Fig. 2 A) and further, the activation of autophagy by Rapamycin alleviated the symptoms of the FES (Fig. 2B). These results demonstrated that autophagy was participated in the mechanism and pathophysiology of FES. However, this mechanism requires further investigation.

Currently, characteristic and therapeutic biomarkers specific for FES remain largely unknown. The therapy of FES mainly contain surgical treatment and a variety of drug treatment, such as steroids, heparin, albumin, low molecular weight dextran [46].Autophagy is a complicated cellular process for degradation and recycling of long-lived proteins and organelles. Autophagy is closely associated with both the pathogenesis and prevention of many human diseases. Thus, considerable therapeutic potential of autophagy attracted more and more attention. Several documents declined the therapeutic potential of autophagy in cancer, neurodegenerative disease, ischemia-Reperfusion Injury and so on [47-49]. These results demonstrated the therapeutic potential of autophagy. Our study found that was participated in the mechanism and pathophysiology of FES (Fig. 3). Thus, we suggest that the modulation of autophagy is a potential biomarker for the assessment of FES risk, as well as serving as novel therapeutic targets.

Taken together, we demonstrated that liquid fat could be used to construct the rat model of FES. Furthermore, we sought the investigated the role of autophagy in FES and found the repression of autophagy in FES. In addition, we found the influence of the restoration of autophagy on FES. In short, our study dissected a way to establish the rat model of FES and 
further, expounded the correlation between autophagy and FES. 


\section{Reference}

1. Mellor, A.; Soni, N. Fat embolism. Anaesthesia 2001, 56, 145-154.

2. Walshe, C.M.; Cooper, J.D.; Kossmann, T.; Hayes, I.; lles, L. Cerebral fat embolism syndrome causing brain death after long-bone fractures and acetazolamide therapy. Critical care and resuscitation : journal of the Australasian Academy of Critical Care Medicine 2007, 9, 184-186.

3. Kosova, E.; Bergmark, B.; Piazza, G. Fat embolism syndrome. Circulation 2015, 131, 317-320.

4. Inoue, H.; Ikeda, N.; Tsuji, A.; Kudo, K.; Hanagama, M.; Nata, M. Pulmonary fat embolization as a diagnostic finding for heat exposure. Legal medicine 2009, 11, 1-3.

5. Miller, S.T. How $i$ treat acute chest syndrome in children with sickle cell disease. Blood 2011, 117, 5297-5305.

6. White, T.; Petrisor, B.A.; Bhandari, M. Prevention of fat embolism syndrome. Injury 2006, 37 Suppl 4, S59-67.

7. Aman, J.; van Koppenhagen, L.; Snoek, A.M.; van der Hoeven, J.G.; van der Lely, A.J. Cerebral fat embolism after bone fractures. Lancet 2015, 386, e16.

8. Ioannou, S.; Vlahadami, I.; Voulgarelis, M. Bone marrow necrosis and fat embolism syndrome presented as conus medullaris syndrome in a patient with primary mediastinal large b-cell lymphoma. Leukemia research 2010, 34, 116-118.

9. Bulger, E.M.; Smith, D.G.; Maier, R.V.; Jurkovich, G.J. Fat embolism syndrome. A 10year review. Archives of surgery 1997, 132, 435-439.

10. Wang, A.Z.; Ma, Q.X.; Zhao, H.J.; Zhou, Q.H.; Jiang, W.; Sun, J.Z. A comparative study of the mortality rate of rats receiving a half lethal dose of fat intravenously: Under general anaesthesia versus under spinal anaesthesia. Injury 2012, 43, 311-314.

11. Takada, M.; Chiba, S.; Nagai, T.; Takeshita, H.; Kanno, S.; Ikawa, T.; Sakamoto, K.; Sagi, M.; Ichiba, K.; Mukai, T. Inflammatory responses to neutral fat and fatty acids in multiple organs in a rat model of fat embolism syndrome. Forensic science international 2015, 254, 126-132.

12. Kim, Y.W.; Kim, H.J.; Choi, S.H.; Cho, B.; Hwangbo, L.; Kim, D.C. Hemorrhage in cerebral fat embolisms in a cat model using triolein dependent on the physical properties of triolein. Japanese journal of radiology 2014, 32, 30-37.

13. Zhou, F.; Ji, J.; Song, Q.; Peng, Z.; Zhang, G.; Wang, Y. Pulmonary fat embolism and related effects during femoral intramedullary surgery: An experimental study in dogs. Experimental and therapeutic medicine 2013, 6, 469-474.

14. Felzemburgh, V.A.; Barbosa, R.C.; Nunes, V.L.; Campos, J.H. Fat embolism in liposuction and intramuscular grafts in rabbits. Acta cirurgica brasileira 2012, 27, 289293.

15. Kaushik, S.; Massey, A.C.; Mizushima, N.; Cuervo, A.M. Constitutive activation of chaperone-mediated autophagy in cells with impaired macroautophagy. Molecular biology of the cell 2008, 19, 2179-2192.

16. Chen, L.M.; Song, T.J.; Xiao, J.H.; Huang, Z.H.; Li, Y.; Lin, T.Y. Tripchlorolide induces autophagy in lung cancer cells by inhibiting the pi3k/akt/mtor pathway and improves cisplatin sensitivity in a549/ddp cells. Oncotarget 2017.

17. Boya, P.; Reggiori, F.; Codogno, P. Emerging regulation and functions of autophagy. 
Nature cell biology 2013, 15, 713-720.

18. Li, R.; Ma, H.; Zhang, X.; Li, C.; Xiong, J.; Lu, T.; Mao, Y.; Dai, J.; Liu, L.; Ding, Z. Impaired autophagosome clearance contributes to local anesthetic bupivacaineinduced myotoxicity in mouse myoblasts. Anesthesiology 2015, 122, 595-605.

19. Dalby, K.N.; Tekedereli, I.; Lopez-Berestein, G.; Ozpolat, B. Targeting the prodeath and prosurvival functions of autophagy as novel therapeutic strategies in cancer. Autophagy 2010, 6, 322-329.

20. Saiki, S.; Sasazawa, Y.; Imamichi, Y.; Kawajiri, S.; Fujimaki, T.; Tanida, I.; Kobayashi, H.; Sato, F.; Sato, S.; Ishikawa, K., et al. Caffeine induces apoptosis by enhancement of autophagy via pi3k/akt/mtor/p70s6k inhibition. Autophagy 2011, 7, 176-187.

21. Rubinsztein, D.C.; Marino, G.; Kroemer, G. Autophagy and aging. Cell 2011, 146, 682695.

22. Nixon, R.A. The role of autophagy in neurodegenerative disease. Nature medicine 2013, 19, 983-997.

23. Kammeyer, R.; Devnani, R.; Mehta, R. Cerebral fat embolism syndrome mimicking thrombotic thrombocytopenic purpura in a patient with hemoglobin sc disease. American journal of hematology 2016, 91, 539-542.

24. Ogunlesi, F.; Heeney, M.M.; Koumbourlis, A.C. Systemic corticosteroids in acute chest syndrome: Friend or foe? Paediatric respiratory reviews 2014, 15, 24-27.

25. Mulay, S.R.; Evan, A.; Anders, H.J. Molecular mechanisms of crystal-related kidney inflammation and injury. Implications for cholesterol embolism, crystalline nephropathies and kidney stone disease. Nephrology, dialysis, transplantation : official publication of the European Dialysis and Transplant Association - European Renal Association 2014, 29, 507-514.

26. Mao, Y.; Luo, W.; Zhang, L.; Wu, W.; Yuan, L.; Xu, H.; Song, J.; Fujiwara, K.; Abe, J.I.; LeMaire, S.A., et al. Sting-irf3 triggers endothelial inflammation in response to free fatty acid-induced mitochondrial damage in diet-induced obesity. Arteriosclerosis, thrombosis, and vascular biology 2017, 37, 920-929.

27. Qian, M.; Fang, X.; Wang, X. Autophagy and inflammation. Clinical and translational medicine 2017, 6, 24.

28. Gros, F. Effects of autophagy on joint inflammation. Joint, bone, spine : revue $d u$ rhumatisme 2017, 84, 129-132.

29. Jia, G.; Cheng, G.; Gangahar, D.M.; Agrawal, D.K. Insulin-like growth factor-1 and tnfalpha regulate autophagy through c-jun n-terminal kinase and akt pathways in human atherosclerotic vascular smooth cells. Immunology and cell biology 2006, 84, 448-454.

30. Shi, P.; Sun, L.L.; Lee, Y.S.; Tu, Y. Electroacupuncture regulates the stress-injury-repair chain of events after cerebral ischemia/reperfusion injury. Neural regeneration research 2017, 12, 925-930.

31. Wang, Y.; Wang, W.; Xu, H.; Sun, Y.; Sun, J.; Jiang, Y.; Yao, J.; Tian, Y. Non-lethal sonodynamic therapy inhibits atherosclerotic plaque progression in apoe-/- mice and attenuates ox-ldl-mediated macrophage impairment by inducing heme oxygenase-1. Cellular physiology and biochemistry : international journal of experimental cellular physiology, biochemistry, and pharmacology 2017, 41, 2432-2446.

32. Liu, S.; Huang, L.; Geng, Y.; He, J.; Chen, X.; Xu, H.; Li, R.; Wang, Y.; Ding, Y.; Liu, X. 
Rapamycin inhibits spermatogenesis by changing the autophagy status through suppressing mechanistic target of Rapamycin-p70s6 kinase in male rats. Molecular medicine reports 2017.

33. Sproule, B.J.; Brady, J.L.; Gilbert, J.A. Studies on the syndrome of fat embolization. Canadian Medical Association journal 1964, 90, 1243-1247.

34. Saigal, R.; Mittal, M.; Kansal, A.; Singh, Y.; Kolar, P.R.; Jain, S. Fat embolism syndrome. The Journal of the Association of Physicians of India 2008, 56, 245-249.

35. Gupta, B.; D'Souza, N.; Sawhney, C.; Farooque, K.; Kumar, A.; Agrawal, P.; Misra, M.C. Analyzing fat embolism syndrome in trauma patients at aiims apex trauma center, new delhi, india. Journal of emergencies, trauma, and shock 2011, 4, 337-341.

36. Blankstein, M.; Byrick, R.J.; Richards, R.R.; Mullen, J.B.; Zdero, R.; Schemitsch, E.H. Pathophysiology of fat embolism: A rabbit model. Journal of orthopaedic trauma 2011, 25, 674-680.

37. Poisner, A.M.; Adler, F.; Uhal, B.; Mclff, T.E.; Schroeppel, J.P.; Mehrer, A.; Herndon, B.L.; Lankachandra, K.M.; Molteni, A. Persistent and progressive pulmonary fibrotic changes in a model of fat embolism. The journal of trauma and acute care surgery 2012, 72, 992-998.

38. Poisner, A.; Herndon, B.; Lankachandra, K.; Likhitsup, A.; Al Hariri, A.; Kesh, S.; Molteni, A. Fat embolism sensitizes rats to a "second hit" with lipopolysaccharide: An animal model of pulmonary fibrosis. The journal of trauma and acute care surgery 2015, 78, 552-557.

39. Mathai, B.J.; Meijer, A.H.; Simonsen, A. Studying autophagy in zebrafish. Cells 2017, 6.

40. He, C.; Klionsky, D.J. Regulation mechanisms and signaling pathways of autophagy. Annual review of genetics 2009, 43, 67-93.

41. Singh, S.B.; Lin, H.C. Autophagy counters Ips-mediated suppression of lysozyme. Innate immunity 2017, 23, 537-545.

42. Zhang, C.; Syed, T.W.; Liu, R.; Yu, J. Role of endoplasmic reticulum stress, autophagy, and inflammation in cardiovascular disease. Frontiers in cardiovascular medicine 2017, 4, 29.

43. Wang, Z.Y.; Liu, J.Y.; Yang, C.B.; Malampati, S.; Huang, Y.Y.; Li, M.X.; Li, M.; Song, J.X. Neuroprotective natural products for the treatment of parkinson's disease by targeting the autophagy-lysosome pathway: A systematic review. Phytotherapy research : PTR 2017, 31, 1119-1127.

44. Yang, J.S.; Lu, C.C.; Kuo, S.C.; Hsu, Y.M.; Tsai, S.C.; Chen, S.Y.; Chen, Y.T.; Lin, Y.J.; Huang, Y.C.; Chen, C.J., et al. Autophagy and its link to type ii diabetes mellitus. BioMedicine 2017, 7, 8.

45. Kania, E.; Roest, G.; Vervliet, T.; Parys, J.B.; Bultynck, G. Ip3 receptor-mediated calcium signaling and its role in autophagy in cancer. Frontiers in oncology 2017, 7, 140.

46. Taviloglu, K.; Yanar, H. Fat embolism syndrome. Surgery today 2007, 37, 5-8.

47. Rubinsztein, D.C.; Gestwicki, J.E.; Murphy, L.O.; Klionsky, D.J. Potential therapeutic applications of autophagy. Nature reviews. Drug discovery 2007, 6, 304-312.

48. Baek, K.H.; Park, J.; Shin, I. Autophagy-regulating small molecules and their 
therapeutic applications. Chemical Society reviews 2012, 41, 3245-3263.

49. Bortnik, S.; Gorski, S.M. Clinical applications of autophagy proteins in cancer: From potential targets to biomarkers. International journal of molecular sciences 2017, 18. 